\title{
Rashba effect induced localization in quantum networks
}

\author{
Dario Bercioux ${ }^{1}$, Michele Governale ${ }^{2}$, Vittorio Cataudella ${ }^{1}$, Vincenzo Marigliano Ramaglia ${ }^{1}$ \\ ${ }^{1}$ Coherentia-INFM and Dipartimento di Scienze Fisiche \\ Università degli studi "Federico II", Napoli, Italy \\ ${ }^{2}$ NEST-INFM and Scuola Normale Superiore, \\ Piazza dei Cavalieri 7, I-56126 Pisa, Italy
}

(Dated: November 15, 2018)

\begin{abstract}
We study a quantum network extending in one-dimension (chain of square loops connected at one vertex) made up of quantum wires with Rashba spin-orbit coupling. We show that the Rashba effect may give rise to an electron localization phenomenon similar to the one induced by magnetic field. This localization effect can be attributed to the spin precession due to the Rashba effect. We present results both for the spectral properties of the infinite chain, and for linear transport through a finite-size chain connected to leads. Furthermore, we study the effect of disorder on the transport properties of this network.
\end{abstract}

PACS numbers: 73.23.-b,73.20.Jc,72.25.-b 
Introduction. It has been recently shown that in a particular class of two-dimensional lattices quantum interference due to the the Aharonov-Bohm effect and to the geometry of the network can induce strong electron localization [1, 2]. In such systems when localization occurs particle motion is confined by destructive interference inside a small portion of the network which is called Aharonov-Bohm (AB) cage. This kind of localization does not rely on disorder [3] but only on quantum-interference and on the geometry of the lattice. There have been several theoretical works addressing different aspects of $\mathrm{AB}$ cages as the effect of disorder and electron-electron interaction [4], interaction induced delocalization [2], and transport [5]. From the experimental side, the AB-cage effect has been demonstrated for superconducting [6] and metallic networks [7] in the so called $\mathcal{T}_{3}$ lattice.

As already stated before, in the AB cages localization is due to interference stemming from the fact that an electron traveling along different paths acquire different phases. It is known that the wavefunction of an electron moving in the presence of Spin-Orbit (SO) coupling acquires quantum phases due to the Aharonov-Casher effect [8, 9, 10, 11, 12, 13]. We now focus on the Rashba SO coupling [14], which is present in semiconductor heterostructures due to lack of inversion symmetry in growth direction. It is usually important in small-gap zinc-blende-type semiconductors, and its strength can be tuned by external gate voltages, as it has been demonstrated experimentally [15, 16, 17].

The question we address in this Letter is whether it is possible to have localization in quantum lattices induced only by the SO coupling without magnetic fields. To answer this question we study the minimal model of a bipartite structure containing nodes with different coordination numbers that with magnetic field exhibits electron localization. This model structure is a linear chain of square loops connected at one vertex (see Fig. 11), which we term diamond chain. We have in mind a realization in a semiconductor heterostructure where the bonds are single-channel quantum wires of length $L$ with Rashba SO coupling. External gates should be present to tune the strength of the SO coupling. This one-dimensional lattice retains the essential features of the more complex $\mathcal{T}_{3}$ networks, allowing for simple (even analytical for the spectrum) solutions.

Model and formalism. Neglecting subband hybridization due to the Rashba effect[18, 19], the Hamiltonian for a single-channel wire along a generic direction $\hat{\gamma}$ in the $x-y$ plane reads

$$
\mathcal{H}=\frac{p_{\gamma}^{2}}{2 m}-\frac{\hbar k_{\mathrm{SO}}}{m} p_{\gamma}(\vec{\sigma} \times \hat{z}) \cdot \hat{\gamma}
$$




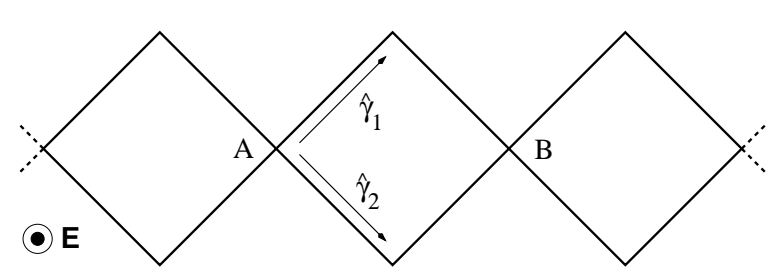

FIG. 1: Schematic view of the diamond chain. The bonds are single-channel quantum wires with SO coupling. In the ideal case all bonds have the same length $L$. The unitary cell contain three nodes (4 wires): one with coordination number 4 and two with coordination number 2.

where $k_{\mathrm{SO}}$ is the $\mathrm{SO}$ coupling strength, and $\vec{\sigma}$ the vector of the Pauli matrices. The SO coupling strength $k_{\mathrm{SO}}$ is related to the spin precession length $L_{\mathrm{SO}}$ by $L_{\mathrm{SO}}=\pi / k_{\mathrm{SO}}$. For InAs quantum wells the spin-precession length ranges from 0.2 to $1 \mu \mathrm{m}$ [15, 16, 17]. These are the characteristic length scales required for the bonds of the network for spin precession to be effective. In order to calculate spectral and transport properties of the network we need to write the wavefunction on a bond (quantum wire) connecting the nodes $\alpha$ and $\beta$, along the direction $\hat{\gamma}_{\alpha \beta}$

$$
\begin{aligned}
\Psi_{\alpha \beta}(r)= & \frac{1}{\sin \left(k l_{\alpha \beta}\right)}\left\{\sin \left[k\left(l_{\alpha \beta}-r\right)\right] e^{i(\vec{\sigma} \times \hat{z}) \cdot \hat{\gamma}_{\alpha \beta} k_{\mathrm{SO}} r} \Psi_{\alpha}\right. \\
& \left.+\sin (k r) e^{i(\vec{\sigma} \times \hat{z}) \cdot \hat{\gamma}_{\alpha \beta} k_{\mathrm{SO}}\left(r-l_{\alpha \beta}\right)} \mathbf{\Psi}_{\beta}\right\}
\end{aligned}
$$

where $k$ is related to the eigen energy by $\epsilon=\frac{\hbar^{2}}{2 m}\left(k^{2}-k_{\mathrm{SO}}^{2}\right)$ [20], $r$ is the coordinate along the bond, and $l_{\alpha \beta}$ the length of the bond. The spinors $\boldsymbol{\Psi}_{\alpha}$ and $\boldsymbol{\Psi}_{\beta}$ are the values of the wavefunction at the nodes $\alpha$ and $\beta$ respectively. The spin precession due to the Rashba effect is described by the exponentials containing Pauli matrices in Eq. (21).

Eq. (2) is the key step to generalize the existing methods to study quantum networks 5, 21] in the presence of Rashba SO coupling. The wavefunction of the whole network is obtained by imposing the continuity of probability current at the nodes. For a generic node $\alpha$ it reads:

$$
\mathbf{M}_{\alpha \alpha} \boldsymbol{\Psi}_{\alpha}+\sum_{\langle\alpha, \beta\rangle} \mathbf{M}_{\alpha \beta} \boldsymbol{\Psi}_{\beta}=0
$$

where

$$
\begin{aligned}
& \mathbf{M}_{\alpha \alpha}=\sum_{\langle\alpha, \beta\rangle} \cot k l_{\alpha \beta} \\
& \mathbf{M}_{\alpha \beta}=-\frac{\exp \left[-i(\vec{\sigma} \times \hat{z}) \cdot \hat{\gamma}_{\alpha \beta} k_{\mathrm{SO}} l_{\alpha \beta}\right]}{\sin k l_{\alpha \beta}} .
\end{aligned}
$$



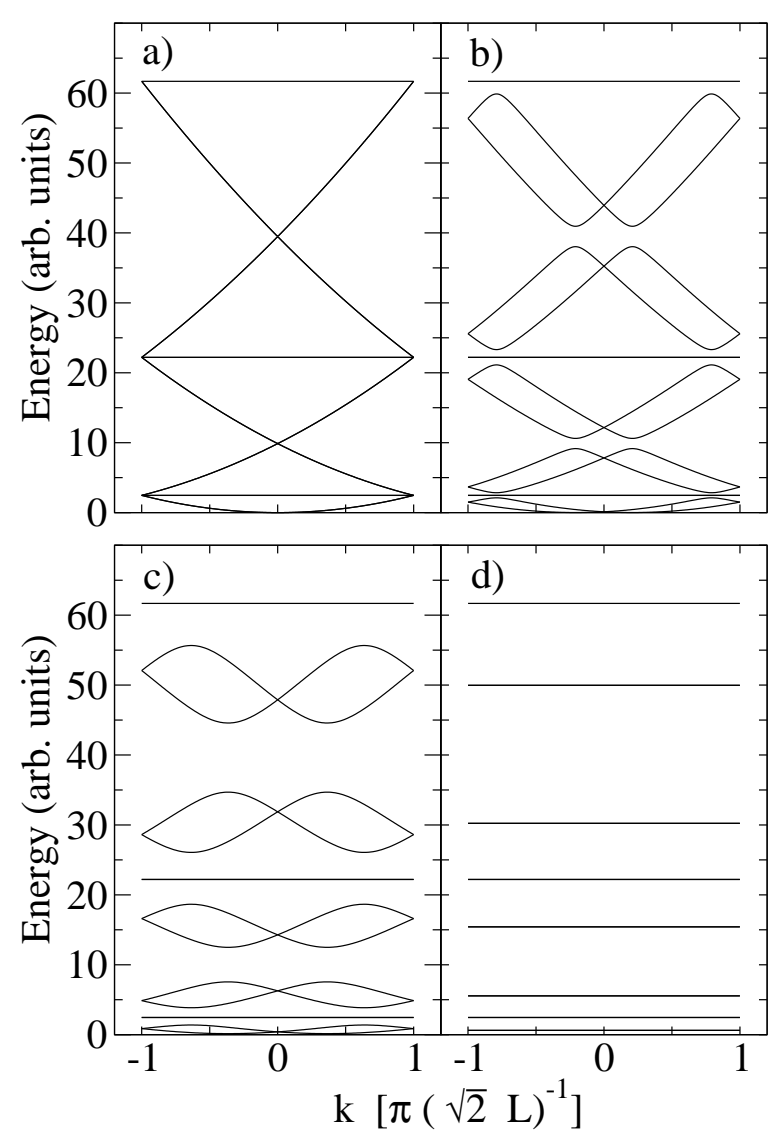

FIG. 2: Spectrum of the diamond chain for different values of the strength of the spin-orbit coupling: a) $k_{\mathrm{SO}} L=0$; b) $k_{\mathrm{SO}} L=0.5$; c) $k_{\mathrm{SO}} L=1.0$; and d) $k_{\mathrm{SO}} L=\pi / 2$.

In Eqs. (34) the sum $\sum_{\langle\alpha, \beta\rangle}$ runs over all nodes $\beta$ which are connected by a bond to the node $\alpha$.

Spectral properties. Now, we apply the method presented above to calculate the spectral properties of the diamond lattice. For an infinite lattice this can be done imposing the Bloch condition on the wavefuction in the unit cell. This straightforward procedure yields for the spectrum the following analytical expressions

$$
\begin{aligned}
\varepsilon_{n}^{(0)}(k)= & \left(\frac{\pi}{2}+n \pi\right)^{2} \\
\varepsilon_{n}^{( \pm)}(k)= & \left\{n \pi+\arccos \left[\frac { 1 } { 2 } \left(2+2 \cos (\sqrt{2} k L) \cos \left(k_{\mathrm{SO}} L\right)^{2}\right.\right.\right. \\
& \left.\left.\left. \pm \sqrt{2} \sin (\sqrt{2} k L) \sin \left(2 k_{\mathrm{SO}} L\right)\right)^{\frac{1}{2}}\right]\right\}^{2}
\end{aligned}
$$

The momentum $k$ is defined in the first Brillouin zone $\left[-\frac{\pi}{\sqrt{2} L}, \frac{\pi}{\sqrt{2} L}\right]$ (notice that the lattice constant is $\sqrt{2} L)$. The spectrum is composed by three kinds of bands. The first one is 
non dispersive: this is a characteristic of every bipartite structures containing nodes with different coordination numbers. The bands \pm are degenerate for zero SO coupling, and are split by it. From Eq. ([6]) it is apparent that these bands become nondispersive for $k_{\mathrm{SO}} L=\left(n+\frac{1}{2}\right) \pi$, being $n$ an integer. This condition can be recast using the spin-precession length as $L=\left(n+\frac{1}{2}\right) L_{\mathrm{SO}}$. For these value of the $\mathrm{SO}$ coupling strength the system becomes localized (as it is indicated by the diverging effective mass). A portion of the spectrum Eqs. (15. 6) is shown in Fig. (21) for increasing values of the SO coupling strength. For zero SO coupling there are no gaps in the spectrum. For finite values of the Rashba coupling the spin degeneracy of the \pm bands is lifted and gaps open in the spectrum. When the SO coupling strength approaches the value $k_{\mathrm{SO}} L=\pi / 2$ the spectrum collapses to a series of non dispersive bands.

The localization for the diamond chain can be understood in terms of interference effects in analogy to the $\mathrm{AB}$ cages. In the case of the $\mathrm{AB}$ effect the phase difference between different paths is due to the enclosed magnetic flux. In the present case the Rashba effect is responsible for it. Consider an electron with spin $|\sigma\rangle$ in A (see Fig. 1). It can reach point $\mathrm{B}$ either via the upper or the lower path. When traveling along the upper path, the spin undergoes a precession first around $\hat{z} \times \hat{\gamma}_{1}$ and then around $\hat{z} \times \hat{\gamma}_{2}$. Hence, the final state in $\mathrm{B}$ is given by $\mathcal{R}_{\hat{\gamma}_{2}} \mathcal{R}_{\hat{\gamma}_{1}}|\sigma\rangle$, where $\mathcal{R}_{\hat{\gamma}}=\exp \left[-i \vec{\sigma} \cdot(\hat{z} \times \hat{\gamma}) k_{\mathrm{SO}} L\right]$. Similarly for propagation along the lower path, the state in $\mathrm{B}$ is $\mathcal{R}_{\hat{\gamma}_{1}} \mathcal{R}_{\hat{\gamma}_{2}}|\sigma\rangle$. Destructive interference occurs when $\left\{\mathcal{R}_{\hat{\gamma}_{1}}, \mathcal{R}_{\hat{\gamma}_{2}}\right\}=0$, being $\{\ldots\}$ the anticommutator. For our setup with $\hat{\gamma}_{1} \cdot \hat{\gamma}_{2}=0$ this condition is fulfilled if $k_{\mathrm{SO}} L=(n+1 / 2) \pi$. A similar analysis can be carried out for more complex structures. In particular, it can be shown that there are bipartite linear chains with a more complex unit cell than the diamond chain that exhibit localization. In analogy to the AB cages, we call the elementary square loop in our structure a Rashba cage.

Transport properties: clean case. In experiments the onset of localization in a quantum network is usually detected by transport measurements. For example, for the AB cages the conductance is suppressed for special values of the magnetic field. To propose a possible experimental verifications of the Rashba-cage effect we now evaluate the linear conductance for a diamond chain of finite length. Furthermore, to show that this localization effect is due to the peculiar geometry of the lattice (bipartite containing nodes with different coordination numbers), we contrast the diamond chain with square ladder, i.e. a chain of square loops connected at two vertices, (see inset of Fig. 3). In the following, we will also 
refer to the latter geometry simply as ladder. We evaluate the conductance making use of the Landauer-Büttiker formalism [22, 23]. We consider a finite piece of lattice connected to semi-infinite leads (with no SO coupling) modeling reservoirs (see inset of Fig. 33). To compute the transmission coefficients we proceed along the lines of Ref. [5]. We inject from the left wire an electron with spin $\sigma= \pm$ along a generic direction, whose corresponding spinors are $\chi_{\sigma}$. The wavefunctions on the external leads are simply

$$
\begin{aligned}
\Psi_{\text {left }}(r) & =e^{i k_{\mathrm{in}} r} \chi_{\sigma}+\sum_{\sigma^{\prime}} r_{\sigma^{\prime} \sigma} e^{-i k_{\mathrm{in}} r} \chi_{\sigma^{\prime}} \\
\Psi_{\text {right }}(r) & =\sum_{\sigma^{\prime}} t_{\sigma^{\prime} \sigma} e^{i k_{\mathrm{in}} r} \chi_{\sigma^{\prime}}
\end{aligned}
$$

where $r$ is the coordinate on the semi-infinite input/output lead, with the origin fixed at the position of the input/output node.

The transmission and reflection coefficients $\left(t_{\sigma^{\prime} \sigma}\right.$ and $r_{\sigma^{\prime} \sigma}$, respectively) can be obtained by solving the linear system of equations arising from the continuity of the probability current at all nodes in the network and of the wavefunction at the input and output nodes. The conditions for the continuity of the probability current at internal nodes are given in Eq. (3). For the external nodes they read

$$
\begin{aligned}
\mathbf{M}_{00} \boldsymbol{\Psi}_{0}+\sum_{\langle 0, \beta\rangle} \mathbf{M}_{0 \beta} \boldsymbol{\Psi}_{\beta} & =-i\left(\chi_{\sigma}-\sum_{\sigma^{\prime}} r_{\sigma^{\prime} \sigma} \chi_{\sigma^{\prime}}\right) \\
\mathbf{M}_{N N} \Psi_{N}+\sum_{\langle N, \beta\rangle} \mathbf{M}_{N \beta} \boldsymbol{\Psi}_{\beta} & =i \sum_{\sigma^{\prime}} t_{\sigma^{\prime} \sigma} \chi_{\sigma^{\prime}}
\end{aligned}
$$

where the injection node is labeled as " 0 " and the output node as " $N$ ". The total transmission coefficient is then simply $|t|^{2}=\sum_{\sigma, \sigma^{\prime}}\left|t_{\sigma^{\prime} \sigma}\right|^{2}$. As it can be seen by inspection of the terms Eq. (41) appearing in the continuity equations (setting $l_{\alpha \beta}=L$ ), all the properties are periodic in $k$ with a periodicity $2 \pi / L$. Furthermore, for the total conductance the period in $k$ is halved, i.e. it is $\pi / L$. Finite temperature or finite voltage will introduce in a natural way an average over $k_{\text {in }}$. For $\operatorname{Max}\left[K_{\mathrm{B}} T, e V\right] \geq K_{\mathrm{B}} T^{*}=\frac{\hbar^{2}}{m} k_{F} \frac{\pi}{L}$, the result of a transport measurement will be the conductance integrated over $k_{\mathrm{in}} \in[0, \pi / L]$, indicated as $\left\langle G\left(k_{\mathrm{SO}} L\right)\right\rangle_{k_{\mathrm{in}}}$. Taking for the Fermi energy of the single-channel wires $10 \mathrm{meV}, \mathrm{m} / \mathrm{m}_{e}=0.042$ for the effective mass (InAs), and $L=1 \mu \mathrm{m}$, yields $T^{*} \approx 7 \mathrm{~K}$.

For a given $k_{\text {in }}$, the conductance has a rich structure that takes into account the complexity of the associate energy spectrum. In particular increasing $k_{\mathrm{SO}}$ gaps open and the 

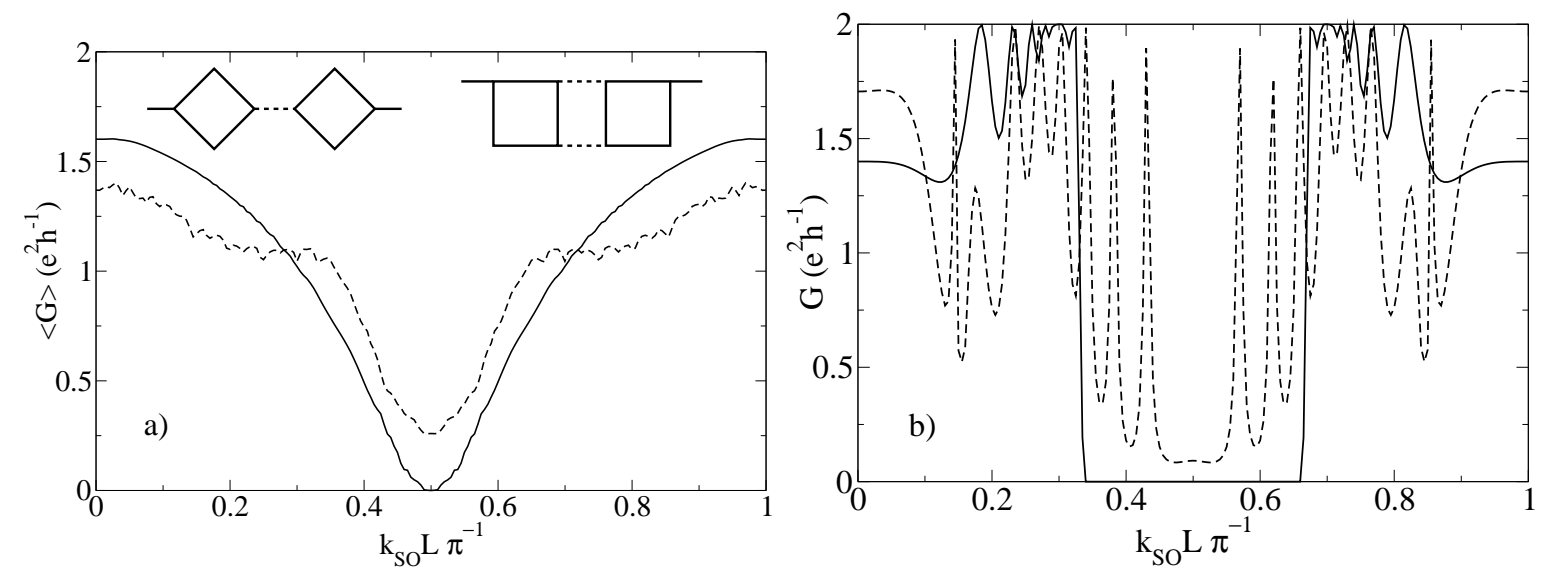

FIG. 3: Panel a): Conductance (averaged over $k_{\text {in }}$ as a function of the spin-orbit coupling strength for the diamond chain (continuous line) and for the ladder (dashed line). The two finite-size systems connected to input/output leads are shown in the inset. The parameters used for the calculation are: 50 elementary loops, $k_{\text {in }}$ uniformly distributed in $[0, \pi / L]$.

Panel b): Conductance as a function of the spin-orbit coupling strength for the diamond chain (continuous line) and for the ladder (dashed line) for a fixed value of $k_{\mathrm{in}}=k_{\mathrm{F}}$. The parameters used for the calculation are: 50 elementary loops, $k_{\mathrm{F}} L=n \pi+2$, being $n$ an integer.

energy of the incoming electrons $\left(\epsilon_{\text {in }}=\frac{\hbar^{2} k_{\text {in }}^{2}}{2 m}\right)$ can enter one of these gaps leading to a vanishing conductance but not to localization [see panel b) of Fig. 3]. In fact, in this case the insulating behavior is due to the absence of available states at the injection energy and not to the localization in space of the electron wavefunction[24]. This effects is not present in $\left\langle G\left(k_{\mathrm{SO}} L\right)\right\rangle_{k_{\mathrm{in}}}$, as the integration over $k_{\text {in }}$ is equivalent to an average over energy. The dependence of the average conductance $\left\langle G\left(k_{\mathrm{SO}} L\right)\right\rangle_{k_{\mathrm{in}}}$ on $k_{\mathrm{SO}}$ is shown in panel a) of Fig. (3) for both the diamond chain and the square ladder. The conductance for both kind of chains has a minimum for $k_{\mathrm{SO}} L=\pi / 2$ due to interference caused by the phase differences induced by the Rashba effect. But due to the existence of the Rashba cages, this minimum reaches zero only for the diamond chain. In panel b) of Fig (3) the conductance for fixed $k_{\text {in }}$ for the two chains is shown: for this choice of parameters, the gap opens only for the diamond chain, while for the ladder a rich interference pattern is present [25].

Transport properties: disordered case. From the studies on the AB cages, we expect the localization induced by the Rashba effect to be robust against disorder only in the bipartite structure containing nodes with different coordination numbers (diamond chain). There 

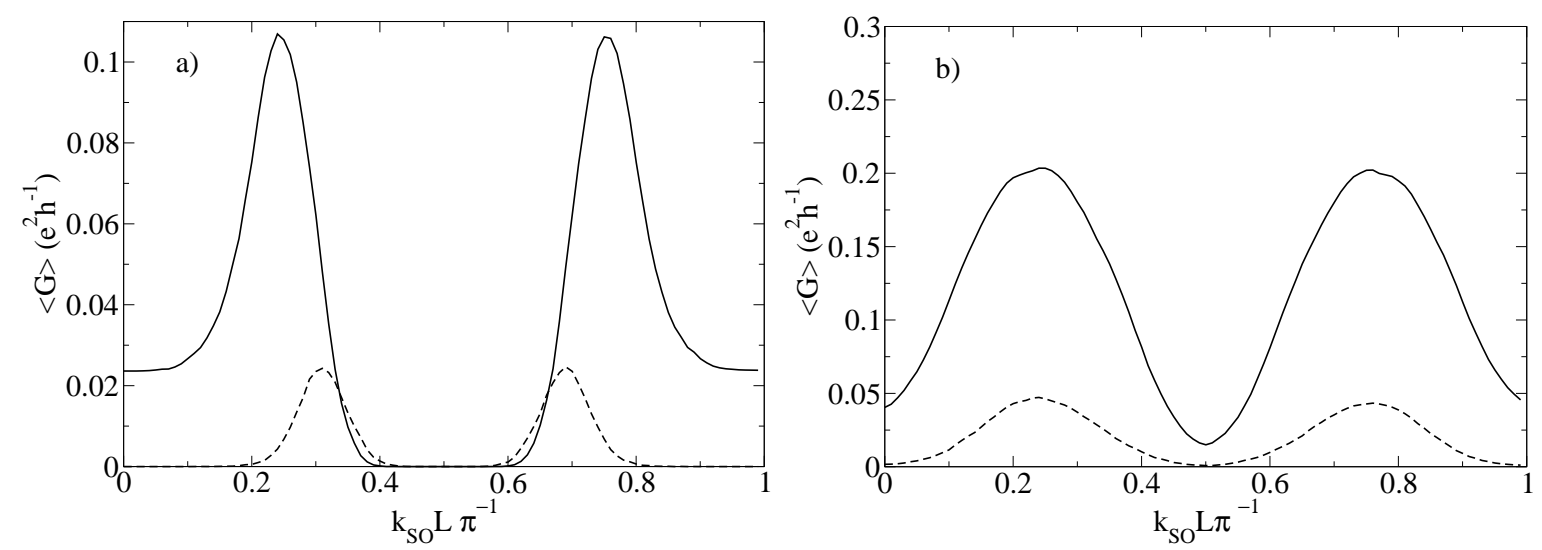

FIG. 4: Conductance (averaged over disorder configurations and over $k_{\text {in }}$ ) plotted as a function of the spin-orbit coupling strength for the diamond chain [panel a)] and the ladder [panel b)]. The two values of the disorder strength used in the calculation are: $\Delta L=0.01 L$ (solid line) and $\Delta L=0.02 L$ (dashed line). Disorder averaging is done over 50 configurations, and $k_{\text {in }}$ is uniformly distributed in $\left[k_{\mathrm{F}}-\pi / 2, k_{\mathrm{F}}+\pi / 2\right]$, with $k_{\mathrm{F}} L=100$. Both systems are composed by 50 elementary loops.

are several kind of disorder that can be considered. Potential disorder along the wires (for example randomly located point-like scatterers) does not lead, in this purely one-dimensional model, to a modification of the phases acquired on a bond by spin-precession but only to a renormalization of the bond transmission. The disorder that is more dangerous for the Rashba-cage effect is a random fluctuation of the length of the bonds (see Ref. [5]), as such length fluctuations induce fluctuations of the phase shifts due to spin-precession. Hence, we consider a model where the length of each bond is randomly distributed in the interval $[L-\Delta L, L+\Delta L]$. The half width of the distribution $\Delta L$ gives the strength of the disorder. In order to clarify if disorder affects the conductance, we average over disorder configurations. This is relevant to experiments, as in a real sample averaging is introduced by the finite phase-coherence length. For intermediate values of disorder $\left(k_{\mathrm{F}} \Delta L \approx 1\right)$ we find that the Rashba-cage effect is still present for the diamond chain, while the periodicity in $k_{\mathrm{SO}}$ is halved for the ladder, as shown in Fig. (41). This latter result can be interpreted as the analogous of the Altshuler-Aharonov-Spivak (AAS) [26] effect induced by the SO coupling. The halving of the oscillation period is due to the enhancement of back-reflection due to interference of pair of paths traveling clockwise and counter-clockwise along a square of the chain (according to weak localization picture). At higher values of disorder the AAS effect 
prevails also in the diamond chain.

Finally, we expect that the results concerning the Rashba-cage effect will not change qualitatively when the wires are multimode if subband hybridization can be neglected, i.e. if

the spin-precession length is much larger than the width of the wires. However, quantitative changes can occur due to scattering between the different modes at the vertices of the network.

Conclusions. We have shown that in quantum network with a particular bipartite geometry (diamond chain) is possible to obtain localization of the electron wavefunction by means of the Rashba effect. This localization shows up both in the spectrum of the infinite chain which becomes nondispersive, and in the transport properties of a finite-size chain connected to leads. Furthermore, transport calculations in the presence of disorder show that in bipartite structure containing nodes with different connectivity this Rashba-cage effect is robust against disorder.

We gratefully acknowledge helpful discussions with G. De Filippis, R. Fazio, D. Frustaglia and A.C. Perroni.

[1] J. Vidal, R. Mosseri, and B. Douçot, Phys. Rev. Lett. 81, 5888 (1998).

[2] J. Vidal, B. Douçot, R. Mosseri, and P. Butaud, Phys. Rev. Lett. 85, 3906 (2000).

[3] P.W. Anderson, Phys. Rev. 109, 1492 (1958).

[4] J. Vidal, P. Butaud, B. Douçot, and R. Mosseri, Phys. Rev. B 64, 155306 (2001)

[5] J. Vidal, G. Montambaux, and B. Douçot, Phys. Rev. B 62, R16294 (2000).

[6] C.C. Abilio, P. Butaud, T. Fournier, B. Pannetier, J. Vidal, S. Tedesco, and B. Dalzotto, Phys. Rev. Lett. 83, 5102 (1999).

[7] C. Naud, G. Faini, and D. Mailly, Phys. Rev. Lett. 86, 5104 (2001).

[8] Y. Aharonov and A. Casher, Phys. Rev. Lett. 53, 319 (1984).

[9] H. Mathur, and A. Douglas Stone, Phys. Rev. Lett. 68, 2964 (1992)

[10] A.V. Balatsky, and B. L. Altshuler, Phys. Rev. Lett. 701678 (1993).

[11] A. G. Aronov and Y. B. Lyanda-Geller, Phys. Rev. Lett. 70, 343 (1993).

[12] J. Splettstößer, M. Governale, and U. Zülicke, Phys. Rev. B 68, 165341 (2003).

[13] D. Frustaglia and K. Richter, cond-mat/0309228. 
[14] E. Rashba, Fiz. Tverd. Tela (Leningrad) 2, 1224 (1960), [Sov. Phys. Solid State 2, 1109 (1960)].

[15] J. Nitta, T. Akazaki, H. Takayanagi, and T. Enoki, Phys. Rev. Lett. 78, 1335 (1997).

[16] T. Schäpers, J. Engels, T. Klocke, M. Hollfelder, and H. Lüth, J. Appl. Phys. 83, 4324 (1998).

[17] D. Grundler, Phys. Rev. Lett. 84, 6074 (2000).

[18] F. Mireles and G. Kirczenow, Phys. Rev. B 64, 24426 (2001).

[19] M. Governale and U. Zülicke, Phys. Rev. B 66, 073311 (2002).

[20] The term in $k_{\mathrm{SO}}^{2}$ can be neglected in realistic situations.

[21] T. Kottos and U. Smilansky, Ann. Phys. (N.Y.) 274, 76 (1999).

[22] R. Landauer, IBM J. Res. Dev. 1, 223 (1957).

[23] M. Buttiker, IBM J. Res. Dev. 32, 317 (1988).

[24] Conductance gaps have been found in quantum wires with Rashba spin-orbit coupling, in the presence of a periodic array of stubs, as a function of the stub length or width in X. F, Wang, P. Vassiolopoulos, and F. M. Peeters, Appl. Phys. Lett. 80, 1400 (2002).

[25] The complexity of the interference pattern is related to the many different interfering paths allowed by the ladder geometry.

[26] B. Altshuler, A. Aharonov, and B. Spivak, Pis'ma Zh. Eksp. Teor. Fiz. 33, 101 (1981), [JEPT Lett. 33, 94, (1981)]. 